\title{
EVALUATION OF AUTONOMIC DYSFUNCTION BY HEART RATE VARIABILITY ANALYSIS IN TYPE 2 DIABETES MELLITUS
}

\author{
PHURPA, SULTANA FERDOUSI*
}

Department of Physiology, Bangabandhu Sheikh Mujib Medical University, Shahbag, Dhaka 1000, Bangladesh. Email: sferdousiratna@gmail.com

Received: 24 September 2016, Revised and Accepted: 12 October 2016

\section{ABSTRACT}

Objective: Cardiac autonomic neuropathy (CAN) is a severe and common, yet highly underdiagnosed, complication of Type 2 diabetes mellitus (T2DM). Subclinical CAN may have reduced heart rate variability (HRV) but normal Ewing battery test. This study was performed to evaluate the importance of 5 minutes HRV for the detection of autonomic dysfunction in T2DM without (CAN-T2DM).

Methods: This cross-sectional observational study was conducted at the Department of Physiology, Bangabandhu Sheikh Mujib Medical University (BSMMU) on 30 recently diagnosed T2DM (RT2DM) and 54 long-term CAN-male T2DM patients (LT2DM), aged 45-55 years, from the Endocrinology Out Patient Department of BSMMU, Dhaka. 30 age and body mass index matched apparently healthy male subjects were control. Ewing battery test was used to rule out CAN positive T2DM. HRV data were recorded by a polyrite-D and analyzed by software. HRV was assessed by time domain method. For statistical analysis, ANOVA and unpaired t-test were used.

Results: Mean RR, standard deviation of NN intervals (SDNN) ( $\mathrm{p}<0.05)$, and root mean square of the successive differences (RMSSD) were significantly $(\mathrm{p}<0.001)$ lower, and mean HR and SDNN/RMSSD were $(\mathrm{p}<0.001)$ significantly higher in LT2DM compared to RT2DM and control. In addition, SDNN was also significantly $(\mathrm{p}<0.05)$ lower in RT2DM than that of control.

Conclusions: Results conclude that autonomic dysfunction may occur in both LT2DM and RT2DM patients without neuropathy and 5 minutes HRV test is an important tool for detecting subclinical CAN.

Keywords: Type 2 diabetes mellitus, Cardiac autonomic neuropathy, Heart rate variability.

(C) 2017 The Authors. Published by Innovare Academic Sciences Pvt Ltd. This is an open access article under the CC BY license (http://creativecommons. org/licenses/by/4. 0/) DOI: http://dx.doi.org/10.22159/ajpcr.2017.v10i1.15344

\section{INTRODUCTION}

Diabetes mellitus (DM) affects 387 million people worldwide with higher prevalence in developing countries [1]. Type 2 DM (T2DM) is the most common form of DM and it is associated with multiple organic complications, including cardiac autonomic neuropathy (CAN) [2,3]. CAN has been extensively studied in T2DM due to its high prevalence, increased risk of cardiovascular mortality, and lack of established treatment until now [4].

CAN may be clinically evident or subclinical which is characterized by functional and reversible neuronal alteration; hence, it is potentially treatable condition if appropriate intervention is provided on time [4-6]. Subclinical stage of CAN could be detected only by test for autonomic nerve function [7].

The almost classical Ewing battery of five cardiovascular reflex tests has been widely used in diagnosing diabetic autonomic neuropathy. Nevertheless, these tests only detect symptomatic or clinical CAN $[4,8]$ which may not be sensitive enough to detect subtle abnormalities in cardiac autonomic nerve activity [9].

Diabetic CAN represents one of only two recommended clinical utilities for heart rate variability (HRV) [10]. HRV has been suggested as one of the diagnostic tests for CAN in a recent statement of the American Diabetes Association [11]. HRV has been referred to as very accurate, noninvasive, and generally reproducible measurement technique, hence, could it be used as a marker for CAN [12].

However, conventionally available autonomic test (Ewing battery test) may appear normal during this stage of CAN [8]. In addition,
Ewing battery test is a lengthy procedure and requires strict patient's cooperation.

Recently, reduced HRV has been recognized as the earliest sign of CAN [9,11,13-17]. 5 minutes HRV test, such as 24 hrs HRV measurement, is a reliable, convenient, and economically practicable technique for detecting autonomic dysfunction [4,9-10,18]. Moreover, only a few studies have been conducted on T2DM patients through 5 minutes HRV analysis to explore the resting autonomic tone.

Duration of diabetes has an important effect on the degree of cardiac autonomic dysfunction associated with DM [19-25]. It may also be found at the time or within a year of diagnosis of T2DM $[26,27]$.

Based on this backdrop, this study was designed to evaluate 5 minutes HRV measures through time domain method in both recently diagnosed and long-term T2DM (RT2DM and LT2DM) patients without CAN, thus, to explore the prospect of 5 minutes HRV test as a technique to detect subclinical CAN in T2DM.

\section{METHODS}

This cross-sectional observational study was carried out from January 2015 to January 2016 at the Department of Physiology, Bangabandhu Sheikh Mujib Medical University (BSMMU), Dhaka. The protocol of the study was approved by the Institutional Review Board of BSMMU. For this study, 30 RT2DM ( $<6$ months of duration of DM) [28] and 54LT2DM (5-10 years of duration of DM) [29] male patients were recruited from the Endocrinology Outpatient Department, BSMMU, after taking written informed consent. All of these patients were only under oral hypoglycemic agents in addition to lifestyle modifications, and none of 
them had any symptom of CAN. All these patients were 45-55 years of age, $18.5-27 \mathrm{Kg} / \mathrm{m}^{2}$ of body mass index (BMI) and $5-10 \%$ of hemoglobin $\mathrm{A} 1 \mathrm{c}(\mathrm{HbA1c}) .30$ age and BMI matched nondiabetic apparently healthy male subjects were enrolled as controls. The patients who had history or were then suffering from: Diabetic retinopathy, hypertension (>140/90 mm Hg), coronary artery diseases, epilepsy, migraine, psychiatric disorders, respiratory disorders, hypo- and hyperthyroidism, consuming drugs with effect on autonomic nervous system function or who were current smokers, yoga practitioners, and athletes were excluded.

The patients who fulfilled the inclusion criteria then underwent a thorough physical examination and Ewing battery test in the Autonomic Lab, Department of Physiology, BSMMU. Based on autonomic nervous system scoring system, the patients who had normal or one borderline finding in these tests were considered to be CAN negative T2DM $[14,29,30]$. However, ones who had $>1$ borderline or $\geq$ abnormal finding were considered CAN positive T2DM patients [11,30,31], and they were excluded from the study. Subsequently, CAN-patients underwent tests for fasting plasma glucose (PPG), 2 hrs PPG (2 hrs $\mathrm{PPG}), \mathrm{HbA1c}$, and plasma creatinine were performed to see glycemic status and to rule out nephropathy.

The finally selected patients were then given instructions to prepare for HRV recording. The night before HRV test: To finish their meal by 09:00 PM, have sound sleep, avoid physical and mental stress, and refrain from drugs having effect on the central nervous system. The morning of HRV test day, they were advised to take light breakfast without coffee or tea.

On HRV test day, the room temperature of the Autonomic Lab was set to $25-28^{\circ} \mathrm{C}$, and the patient was seated in the lab for 15 minutes before HRV recording allowing him to adjust with the lab environment. Then, the patient was requested to lie on the examination bed in supine position and to rest for 5 minutes more. After that, electrocardiogram was recorded on lead II for 5 minutes, by RMS multichannel polyrite-D, from which time domain measures of HRV was analyzed by polyrite software.

Data were expressed as mean and standard error of mean. For statistical analysis, one-way ANOVA and independent sample t-test were used as applicable. SPSS version 16 was used for data analysis. $\mathrm{p}<0.05$ was taken as statistically significant level.

\section{RESULTS}

General characteristics of all subjects are presented in Table 1. BMI of RT2DM was significantly $(\mathrm{p}<0.05)$ higher than that of control and LT2DM, and pulse rate was significantly $(\mathrm{p}<0.05)$ increased in longterm DM compared to control and recently diagnosed DM. There was

Table 1: General characteristics of different groups (n=114)

\begin{tabular}{llll}
\hline Parameters & Control (C) & RT2DM & LT2DM \\
\hline Age (years) & $48.5 \pm 0.50$ & $48.54 \pm 0.60$ & $49.78 \pm 0.38$ \\
BMI (kg/m ${ }^{2}$ ) & $22.89 \pm 0.50$ & $25.03 \pm 0.74^{*}$ & $23.17 \pm 0.19$ \\
Pulse rate (beats/min) & $77.03 \pm 0.80$ & $77.69 \pm 0.73$ & $80.48 \pm 0.58^{\#}$ \\
SBP (mm Hg) & $125.33 \pm 1.04$ & $125.82 \pm 1.54$ & $126.02 \pm 1.12$ \\
DBP (mm Hg) & $77.0 \pm 0.88$ & $78.65 \pm 0.81$ & $78.61 \pm 0.69$ \\
FPG (mmol/L) & $4.96 \pm 0.14$ & $8.16 \pm 0.26^{¥}$ & $7.67 \pm 0.14^{¥}$ \\
2-hrs PPG (mmol/L) & - & $11.30 \pm 0.31$ & $12.30 \pm 0.23$ \\
HbA1c (\%) & - & $8.11 \pm 0.21^{\psi}$ & $7.18 \pm 0.07$ \\
\hline
\end{tabular}

${ }^{*} \mathrm{p}<0.001$ (RT2DM vs. LT2DM, C); ${ }^{*}<<0.05$ (LT2DM vs. RT2DM, C);

${ }^{*} \mathrm{p}<0.05$ (RT2DM, LT2DM vs. C); $p<0.05$ (LT2DM vs. RT2DM); ${ }^{4}<<0.001$ (RT2DM

vs. L2TDM). Data were expressed as mean \pm SEM. ANOVA and independent

sample t-test were used. BMI: Body mass index, SBP: Systolic blood pressure,

DBP: Diastolic blood pressure, RT2DM: Recently diagnosed Type 2 diabetes mellitus, LT2DM: Long-term Type 2 diabetes mellitus, SEM: Standard error of mean, FPG: Fasting plasma glucose, PPG: Plasma glucose,

HbA1c: Hemoglobin A1c a significant decrease in mean RR, root mean square of the successive differences (RMSSD), and standard deviation of NN intervals (SDNN) $(p<0.001)$ in LT2DM compared to control and recently diagnosed ones, whereas the difference between the latter two groups was statistically not significant, except SDNN, which was also significantly lower in recently diagnosed DM compared to control (Figs. 1-3). Mean HR and SDNN/RMSSD $(\mathrm{p}<0.001)$ were significantly higher in LT2DM compared to control and RT2DM, and these values in the latter two groups were almost similar (Figs. 4 and 5).

\section{DISCUSSION}

In this study, SDNN, which reflects overall variability, derived from short-term HRV analysis was significantly reduced in RT2DM patients compared to controls and it was markedly reduced in DM patients with longer duration. Similar result was reported in previous studies which stated that there was decrease in overall variability of HR [9,24,32-34]. This demonstrates reduced overall HRV but with the greater degree of impairment in patients with longer duration of disease.

Again, notable decrease in mean RR, RMSSD, and variance in DM patients with longer duration confirmed the previous findings made by other researchers $[9,24,32-34]$. These results suggest marked impairment of

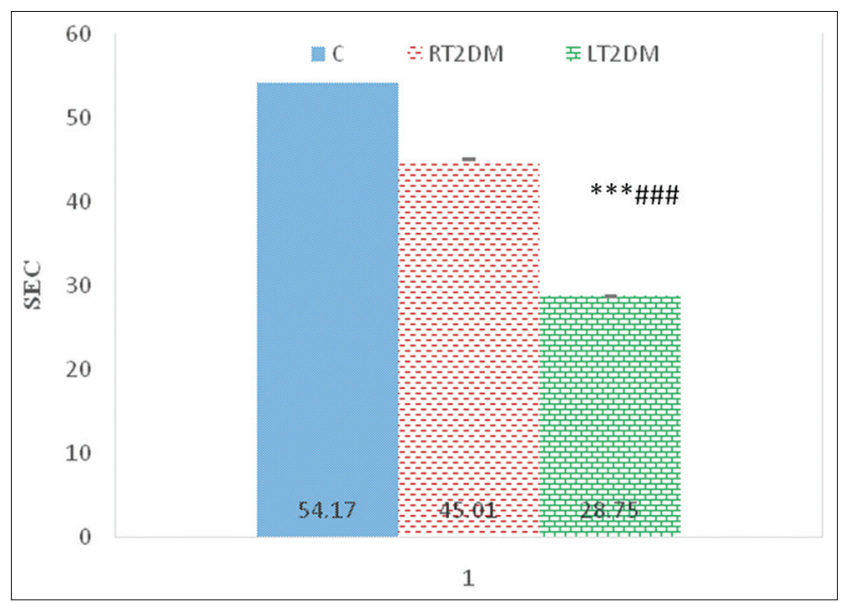

Fig. 1: Mean \pm standard error of mean RR interval in different groups. RT2DM: Recently diagnosed Type 2 diabetes mellitus, LT2DM: Long-term Type 2 diabetes mellitus, C: Control, ***p $<0.001$, LT2DM versus C; \#\#\# $<<0.001$, LT2DM versus RT2DM

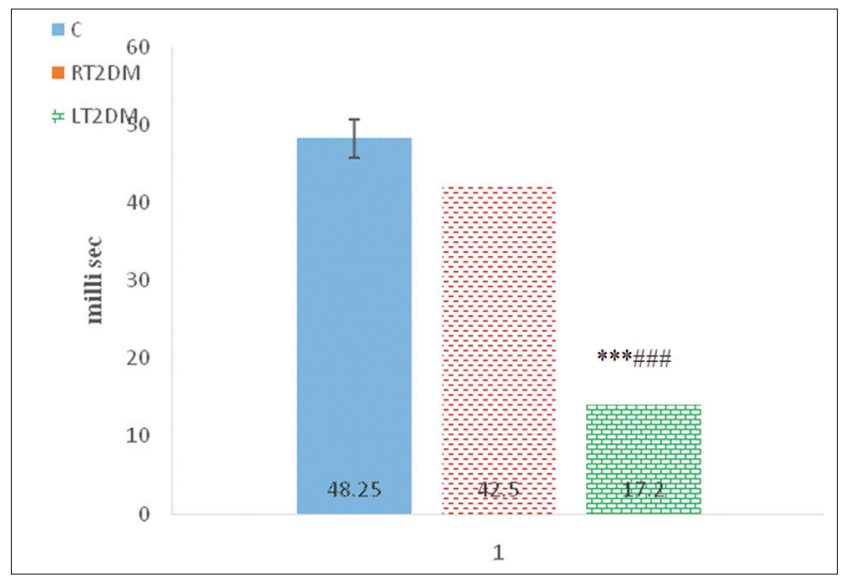

Fig. 2: Mean \pm standard error of mean root mean square of the successive differences in different groups. RT2DM: Recently

diagnosed Type 2 diabetes mellitus, LT2DM: Long-term Type 2 diabetes mellitus, C: Control, ${ }^{* * *} \mathbf{p}<0.001$, LT2DM versus C; $\# \#$ p $<0.001$, LT2DM versus RT2DM 


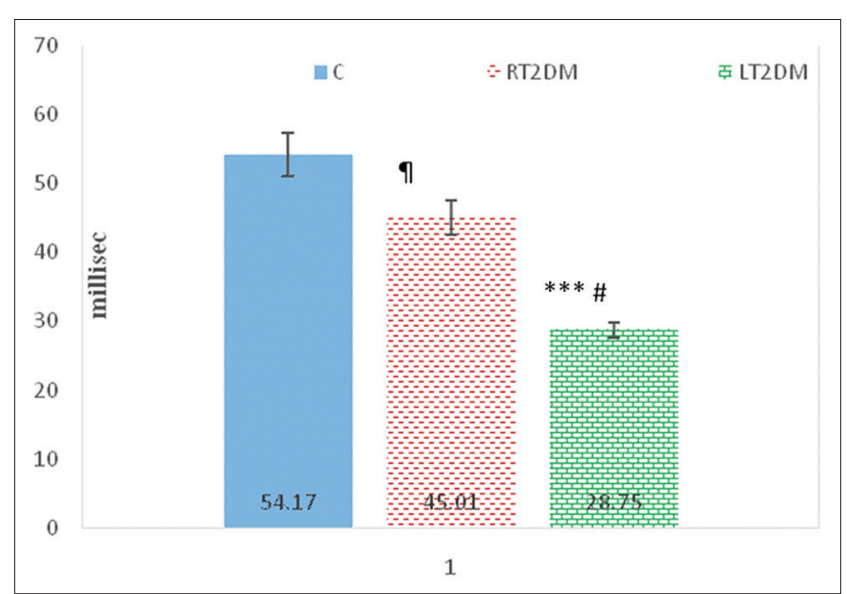

Fig. 3: Mean \pm standard error of mean standard deviation of NN intervals in different groups. RT2DM: Recently diagnosed Type 2 diabetes mellitus, LT2DM: Long-term Type 2 diabetes mellitus, C: Control, ${ }^{* * *} \mathbf{p}<0.001$, LT2DM versus C; \#p $<0.05$, LT2DM versus RT2DM, p<0.01, RT2DM versus C

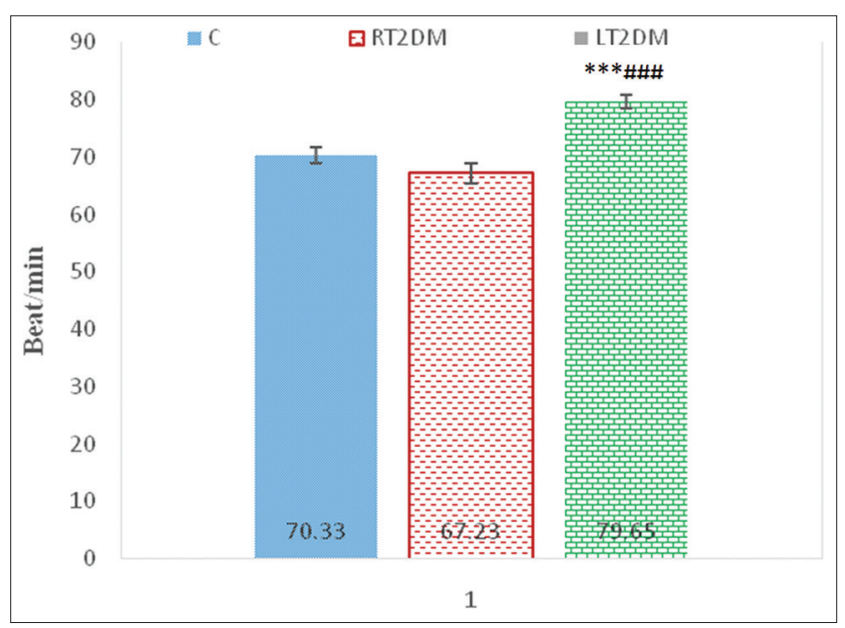

Fig. 4: Mean \pm standard error of mean HR in different groups. RT2DM: Recently diagnosed Type 2 diabetes mellitus, LT2DM: Long-term Type 2 diabetes mellitus, C: Control, ${ }^{* * *} \mathbf{p}<\mathbf{0 . 0 0 1}$, LT2DM versus $C$, \#\#\#p<0.05, LT2DM versus RT2DM

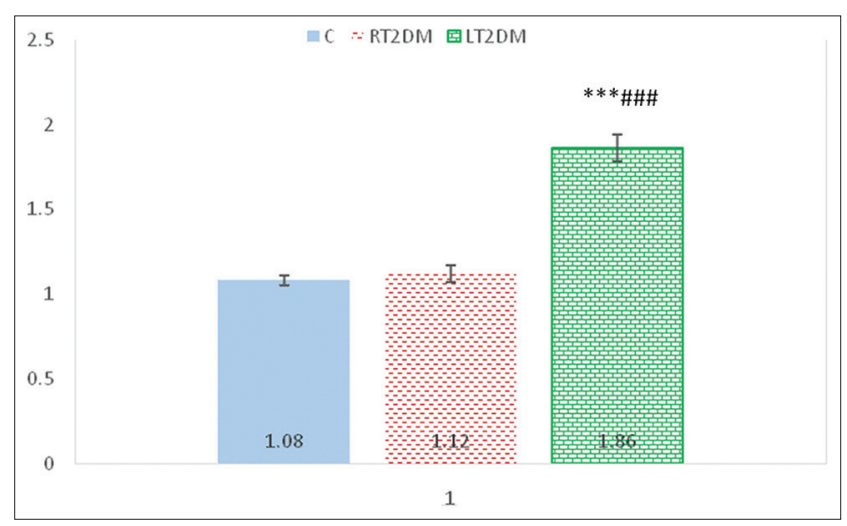

Fig. 5: Mean \pm standard error of mean standard deviation of NN intervals/root mean square of the successive differences in different groups. RT2DM: Recently diagnosed Type 2 diabetes mellitus, LT2DM: Long-term Type 2 diabetes mellitus, C: Control, ${ }^{* * *} \mathbf{p}<0.001$, LT2DM versus $C$, ${ }^{\# \# \# p<0.05, ~ L T 2 D M ~ v e r s u s ~ R T 2 D M ~}$ parasympathetic nerve function impairment and small involvement of sympathetic component in them. This is consistent with the statement, made by previous studies, that parasympathetic nerve discharge is decreased even at rest [34]. Nonetheless, these values in recently diagnosed group were almost similar to those of control indicating little effect in these patients as opposed to previous publication [32]. This might be due to the fact that unlike in this study, the T2DM patients with $<2$ years duration of DM was taken as RT2DM. Moreover, duration of DM has been said to have a significant association with the degree of autonomic dysfunction in many previous studies [19-25].

Mean HR, a representation of interaction between sympathetic and parasympathetic modulation of the heart, with predominant parasympathetic tone at rest [10], showed significant increment in LT2DM compared to control and recently diagnosed DM. This finding is consistent to previous finding [33]. Decreased parasympathetic nerve function might be responsible for increased HR as stated by the previous study [35]. This result suggests increased sympathetic nerve activity in LT2DM patients.

SDNN/RMSSD in literature has been used as a marker of autonomic balance [36], and it was significantly increased in LT2DM Group, denoting autonomic imbalance in LT2DM patients characterized by sympathetic overdrive.

It is important to note that all these previous studies, except the one conducted by Tarvainen et al,, be it on LT2DM or RT2DM were done without ruling out the presence of CAN based on Ewing battery test [24].

The pathogenesis of CAN is still unclear, yet, hyperglycemia and hyperinsulinemia or insulin resistance induced neuronal damage seem to be the common area of focus among many researchers [26,36-38]. At early stage of CAN or during subclinical CAN, vagus nerve is the first to undergo damage resulting into sympathetic overactivity during which it may manifest with resting tachycardia and exercise intolerance, and abnormal findings in Ewing battery test [4]. During later stage, sympathetic denervation ensues, which may manifest with orthostatic hypotension [4]

In a nutshell, reduced HRV in both Groups of DM even in the absence of clinically evident autonomic neuropathy suggests the presence of autonomic dysfunction in its early stage. Moreover, there is greater impairment of parasympathetic component resulting into autonomic imbalance featured by sympathetic overdrive which is more evident in DM with longer duration.

The results in RT2DM provide a hint toward the initiation of autonomic disorder associated with the earliest stage of diabetes as said by previous studies that cardiac autonomic dysfunction could be present at the time of diagnosis or within a year of diagnosis of T2DM [26,27]. Nevertheless, the time period over which the subclinical CAN turns into clinical CAN or develops abnormal Ewing battery test is still uncertain [4]. Notwithstanding, subclinical CAN may manifest with reduced HRV, which is often undetected by the Ewing battery test, but detected HRV analysis $[4,8]$.

Thus, it is noteworthy that all these evidence of autonomic dysfunction in its earliest stage could be traced only by short-term HRV.

\section{CONCLUSIONS}

Results of this study concluded that cardiac autonomic dysfunction was present in LT2DM patients without CAN (based on conventional autonomic test) characterized by predominant parasympathetic nerve function impairment and sympathetic overactivity. Moreover, subtle cardiac autonomic dysfunction occurs in RT2DM without CAN.

Hence, short-term HRV test is an essential technique for detection subclinical stage of CAN. 


\section{ACKNOWLEDGMENT}

The authors of this study are thankful to the university grant commission of Bangladesh, for providing a research grant to support this study.

\section{REFERENCES}

1. International Diabetes Federation. South-East Asia, Bangladesh, Brussels, (Belgium): International Diabetes Federation; 2014. Available from: http://www.idf.org sea. [Last cited on 2015 Oct 03]

2. Ahmed KA, Muniandy S, Ismail IS. Type 2 diabetes and vascular complications: A pathophysiologic view. Biomed Res 2010;21(2):147-55.

3. Tiwari BK, Pandey KB, Abidi AB, Rizvi SI. Markers of oxidative stress during diabetes mellitus. J Biomark 2013;2013:378790.

4. Dimitropoulos G, Tahrani AA, Stevens MJ. Cardiac autonomic neuropathy in patients with diabetes mellitus. World J Diabetes 2014;5(1):17-39.

5. Rolim LC, Sá JR, Chacra AR, Dib SA. Diabetic cardiovascular autonomic neuropathy: Risk factors, clinical impact and early diagnosis. Arq Bras Cardiol 2008;90(4):e24-31.

6. Prakash SB, Ryaz AS, Itagi V, Dixit P. Heart rate variability as a tool to detect cardiac autonomic neuropathy in Type 2 diabetic patients. Pariprex Indian J Res 2015;4(9):10-1.

7. Asbury AK, Porte D Jr. Consensus statement on standardized measures in diabetic neuropathy. Diabetes Care 1995;18 Suppl 1:59-82.

8. Khandoker AH, Jelinek HF, Moritani T, Palaniswami M. Association of cardiac autonomic neuropathy with alteration of sympathovagal balance through heart rate variability analysis. Med Eng Phys 2010;32(2):161-7.

9. Deepak A, Aithal K, Khode VH, Nallulwar SC. Short term heart rate variability for early assessment of autonomic neuropathy in patients with Type 2 diabetes mellitus: A comparative cross-sectional study. Ann Niger Med 2014;8(1):4-7.

10. Heart rate variability: Standards of measurement, physiological interpretation and clinical use. Task Force of the European Society of Cardiology and the North American Society of Pacing and Electrophysiology. Circulation 1996;93(5):1043-65.

11. Balcioglu S, Arslan U, Türkoglu S, Ozdemir M, Cengel A. Heart rate variability and heart rate turbulence in patients with Type 2 diabetes mellitus with versus without cardiac autonomic neuropathy. Am J Cardiol 2007;100(5):890-3.

12. Al-Hazimi A, Al-Ama N, Syiamic A, Qosti R, Abdel-Galil K. Timedomain analysis of heart rate variability in diabetic patients with and without autonomic neuropathy. Ann Saudi Med 2002;22(5-6):400-3

13. Ziegler D. Diabetic cardiovascular autonomic neuropathy: Prognosis, diagnosis and treatment. Diabetes Metab Rev 1994;10(4):339-83.

14. Karamitsos DT, Didangelos TP, Athyros VG, Kontopoulos AG. The natural history of recently diagnosed autonomic neuropathy over a period of 2 years. Diabetes Res Clin Pract 1998;42(1):55-63.

15. Vinik AI, Maser RE, Mitchell BD, Freeman R. Diabetic autonomic neuropathy. Diabetes Care 2003;26(5):1553-79.

16. Stein PK, Barzilay JI, Domitrovich PP, Chaves PM, Gottdiener JS, Heckbert SR, et al. The relationship of heart rate and heart rate variability to non-diabetic fasting glucose levels and the metabolic syndrome: The Cardiovascular Health Study. Diabet Med 2007;24(8):855-63.

17. Pop-Busui R. Cardiac autonomic neuropathy in diabetes: A clinical perspective. Diabetes Care 2010;33(2):434-41.

18. Sacre JW, Jellis CL, Marwick TH, Coombes JS. Reliability of heart rate variability in patients with Type 2 diabetes mellitus. Diabet Med 2012;29(7):e33-40.

19. Ziegler D, Dannehl K, Volksw D, Mühlen H, Spüler M, Gries FA. Prevalence of cardiovascular autonomic dysfunction assessed by spectral analysis and standard tests of heart-rate variation in newly diagnosed IDDM patients. Diabetes Care 1992;15(7):908-11.

20. Ko SH, Kwon HS, Lee JM, Kim SR, Cho JH, Yoo KD, et al. Cardiovascular autonomic neuropathy in patients with Type 2 diabetes mellitus. J Korean Diabetes Assoc 2006;30(3):226-35.

21. Alam KM, Begum N, Molla HM, Begum S. Sympathetic nerve function status in Type 2 diabetes mellitus: Relationship with glycaemic status and duration. J Med Sci Res 2008;11(1):23-30.

22. Cabezas-Cerrato J, Gonzalez-Quintela A, Perez-Rodriguez M, Calle A, Faure-Noguera E, Vazquez-Garcia JA; Neuropathy Spanish study group of the Spanish Diabetes Society (SDS). Combination of cardiorespiratory reflex parameters and heart rate variability power spectrum analysis for early diagnosis of diabetic cardiac autonomic neuropathy. Diabetes Metab 2009;35:305-11.

23. Spallone V, Ziegler D, Freeman R, Bernardi L, Frontoni S, PopBusui $\mathrm{R}$, et al. Cardiovascular autonomic neuropathy in diabetes: Clinical impact, assessment, diagnosis, and management. Diabetes Metab Res Rev 2011;27(7):639-53.

24. Tarvainen MP, Laitinen TP, Lipponen JA, Cornforth DJ, Jelinek HF. Cardiac autonomic dysfunction in Type 2 diabetes - Effect of hyperglycemia and disease duration. Front Endocrinol (Lausanne) 2014;5:130.

25. Hussain A, Rahim MA, Azad Khan AK, Ali SM, Vaaler S. Type 2 diabetes in rural and urban population: Diverse prevalence and associated risk factors in Bangladesh. Diabet Med 2005;22(7):931-6.

26. Kuehl M, Stevens MJ. Cardiovascular autonomic neuropathies as complications of diabetes mellitus. Nat Rev Endocrinol 2012;8:405-16.

27. Pfeifer MA, Weinberg CR, Cook DL, Reenan A, Halter JB, Ensinck JW, et al. Autonomic neural dysfunction in recently diagnosed diabetic subjects. Diabetes Care 1984;7(5):447-53.

28. Lieb DC, Parson HK, Mamikunian G, Vinik AI. Cardiac autonomic imbalance in newly diagnosed and established diabetes is associated with markers of adipose tissue inflammation. Exp Diabetes Res 2012;2012:878760.

29. McDaid EA, Monaghan B, Parker AI, Hayes JR, Allen JA. Peripheral autonomic impairment in patients newly diagnosed with Type II diabetes. Diabetes Care 1994;17(12):1422-7.

30. Bannister SR, Mathias CJ. A Text Book of Clinical Disorders of the Autonomic Nervous System. $3^{\text {rd }}$ ed. New York: Oxford University Press; 1992.

31. Ewing DJ, Martyn CN, Young RJ, Clarke BF. The value of cardiovascular autonomic function tests: 10 years experience in diabetes. Diabetes Care 1985;8(5):491-8.

32. Michel-Chávez A, Estañol B, Gien-López JA, Robles-Cabrera A, Huitrado-Duarte ME, Moreno-Morales R, et al. Heart rate and systolic blood pressure variability on recently diagnosed diabetics. Arq Bras Cardiol 2015;105(3):276-84.

33. Tale S, Sontakke TR. Time-Frequency Analysis of Heart Rate Variability Signal in Prognosis of Type 2 Diabetic Autonomic Neuropathy. Singapore: International Conference on Biomedical Engineering and Technology; 2011.

34. Pramodh V, Kumar MP, Prasad BA. Heart rate variability in Type 2 diabetics. Int J Biomed Res 2014;5(5):364-7.

35. Wang H, Huang S. SDNN/RMSSD as a surrogate for LH/HF: A revised investigation. Model Simul Eng 2012;2012:8.

36. Giacco F, Brownlee M. Oxidative stress and diabetic complications. Circ Res 2010;107(9):1058-70.

37. de M Bandeira S, da Fonseca LJ, da S Guedes G, Rabelo LA, Goulart MO, Vasconcelos SM. Oxidative stress as an underlying contributor in the development of chronic complications in diabetes mellitus. Int J Mol Sci 2013;14(2):3265-84.

38. Manzella D, Paolisso G. Cardiac autonomic activity and Type II diabetes mellitus. Clin Sci (Lond) 2005;108(2):93-9. 